\title{
JOINT SURFACE DEFECTS: CLINICAL COURSE AND CELLULAR RESPONSE IN SPONTANEOUS AND EXPERIMENTAL LESIONS
}

\author{
F. Dell'accio ${ }^{1 *}$, and T.L. Vincent ${ }^{2}$ \\ ${ }^{1}$ Centre of Experimental Medicine and Rheumatology, William Harvey Research Institute \\ Barts and The London, Queen Mary's School of Medicine and Dentistry, London, U.K. \\ ${ }^{2}$ Kennedy Institute of Rheumatology, London, U.K.
}

\begin{abstract}
Joint surface defects (JSD) involving the articular cartilage and the subchondral bone are a common clinical problem in rheumatology and orthopaedics. The recent availability of accurate imaging for diagnosis and efficacious therapeutic options has stirred new interest in their natural history and biology. The evidence that some of these lesions can heal spontaneously whereas others precipitate osteoarthritis has raised important questions as to which lesions should be treated, when, and how. Evidence of repair of some of these lesions has also stimulated research into which factors contribute to successful healing and which ones determine chronic evolution and development of osteoarthritis (OA). Older anatomical observations, together with novel molecular tools and experimental models, have revealed a complex cellular and molecular response of cartilage to focal defects, which could explain differences in healing responses between individuals, and may provide clues to stimulating intrinsic tissue repair. In the first part of this review we will discuss clinical aspects of these lesions in the patient, with particular emphasis on their biology and natural history. In the second part we will summarize the data coming from in vitro and in vivo models of cartilage injury and regeneration, focussing on the molecular control of cartilage homeostasis after creation of cartilage surface defects.
\end{abstract}

Keywords: Joint surface defect, cartilage biology, cartilage repair, regeneration, osteoarthritis, Wnt, FGF, animal models.

\footnotetext{
*Address for correspondence:

F. Dell'accio

Centre of Experimental Medicine and Rheumatology

William Harvey Research Institute

Barts and The London, Queen Mary's School of Medicine and Dentistry

II floor John Vane Building

Charterhouse Square

London EC1M 6BQ, U.K.

Telephone Number: +44 (0)20 78828204

E-mail f.dellaccio@qmul.ac.uk
}

\section{Prevalence and aetiology}

Joint surface defects (JSDs) are focal lesions of the articular cartilage. They are very common, being reported in about $20 \%$ of all arthroscopic procedures (Curl et al., 1997; Hjelle et al., 2002). They are clinically important as they can be symptomatic and disabling, with pain and/ or locking of the joint, and can predispose to further cartilage loss and development of osteoarthritis (OA) (Ding et al., 2008). Awareness of these lesions has increased with the development of non-invasive imaging for diagnosis (MRI), and the recent emergence of efficacious therapies for cartilage repair.

Chondral lesions vary greatly in their morphology and topography, and this variation influences their outcome and clinical manifestations. Broadly speaking, JSD can be superficial, partial thickness cartilage defects, which do not involve the subchondral bone, and full thickness lesions which cross the osteochondral junction. Superficial cartilage defects rarely represent a clinical problem since they are usually asymptomatic and there is little evidence to indicate that they predispose to OA (Messner and Maletius, 1996; Shelbourne et al., 2003; Ding et al., 2005a; Smith et al., 2005). Indeed, chondral surgery, such as autologous chondrocyte implantation or microfracture, is indicated for full thickness, chronic, symptomatic, chondral or osteochondral defects (Brittberg et al., 1994; Peterson et al., 2000; Brittberg et al., 2003; Smith et al., 2005; Brittberg, 2008). This distinction may be somewhat artificial because longitudinal studies have shown that full thickness lesions can become partial thickness and vice versa (Cicuttini et al., 2005; Nakamura et al., 2008).

Trauma has traditionally been regarded as the most important aetiological factor in the development of focal chondral or osteochondral defects (Morscher, 1979). However, in a large study of 1000 consecutive arthroscopies, $39 \%$ of patients with a focal defect in their knee cartilage failed to remember a previous traumatic episode to their joint (Hjelle et al., 2002). Moreover, up to $43 \%$ of healthy subjects without a family history of OA have knee chondral lesions as evaluated by MRI (Ding et al., 2005b). These data point to the fact that chondral or osteochondral defects are more common than previously thought, are not necessarily of traumatic origin, and need not be symptomatic. Therefore, the ability to identify which lesions become progressive and require intervention is of paramount importance.

\section{Natural history}

Over 250 years ago Hunter stated that "If we consult the standard Chirurgical Writers from Hippocrates down to 
the present Age, we shall find, that an ulcerated Cartilage is universally allowed to be a very troublesome disease and when destroyed, it is never recovered." (Hunter, 1743). This statement still probably stands true for symptomatic defects that have acquired a chronic course, such as those that reach the attention of the doctor or the surgeon. Until a few years ago, this was also assumed to be true for all JSDs, and was linked to the observation that the risk of developing OA by the age of 65 was $13 \%$ in individuals with a history of trauma and $6 \%$ in those without a history of trauma (Gelber et al., 2000). Clearly, many factors other than JSDs account for the modestly higher relative risk of $\mathrm{OA}$ in patients with previous knee trauma, including lesions to ligaments and menisci.

In 1996, Messner and Maletius (1996) reported that 22 out of 28 young athletes with an isolated chondral injury in a weight bearing part of their knee diagnosed by arthroscopy had good or excellent knee function at 14 years of follow up as evaluated clinically and radiographically. No specific treatment had been preformed except, in 3 cases, Pridie drilling (similar to microfracture) and occasional debridement. 21 patients were able to return to pre-injury level sports activities after trauma and arthroscopy. The overall level of activity decreased at the later time points of follow up (14 years) in relation to a decline in engagement in competitive team sports, but since there was no control group it is impossible to determine whether this declined was influenced by the injury. Although at the end of follow-up 12 patients had some radiographic joint space reduction, no control group was included and therefore we do not know whether joint space reduction would have occurred in the absence of a JSD, particularly since this cohort was composed of professional athletes (Messner and Maletius, 1996). There was no significant difference with the contralateral knee in terms of signs of OA. In this study, all patients had an isolated Outerbridge grade 2 (most cases) or grade 3 chondral defect (diameter $>1 \mathrm{~cm}$ ) at presentation, without any damage to other joint structures including menisci, ligaments, and the remaining cartilage. The Outerbridge scale categorises superficial defects as grade I; deeper lesions not reaching the subchondral bone as grade II; fissuring to the level of subchondral bone as grade III, and lesions exposing the subchondral bone as grade IV. No patient had instability, or a previous history of knee surgery. What we learn from this study is that isolated chondral or osteochondral lesions, in young active patients, in otherwise healthy knees, have a favourable natural outcome leading to long term functional restoration. We do not know whether (good) structural repair is required for functional outcome or whether these lesions become asymptomatic or repair with scar tissue. In either case, such a good outcome after 14 years follow up in $78 \%$ of lesions suggest, at least, that an aggressive approach to treatment of all such lesions is not justified.

The above study followed individuals with focal chondral defects in otherwise normal knees. However, the majority of symptomatic patients who have chondral defects detected on arthroscopy, have additional joint pathology, including lesions affecting the menisci or ligaments (Brittberg, 2008). In a longitudinal study, Shelbourne et al. (2003) asked the question whether the presence of a chondral injury detected in young athletes undergoing ACL reconstruction modifies the clinical outcome and requires chondral repair. In this study the clinical outcome of patients that had either a single chondral or osteochondral injury at the time of arthroscopy, was compared with that of age and sex matched patients who underwent ACL reconstruction but had no chondral injury. The cartilage injury was left untreated, and the clinical outcome was monitored for 8.7 years, clinically and radiographically. Although the symptoms of patients with a chondral injury were slightly more severe, $79 \%$ of them returned to pre-injury levels of sports activities involving jumping, twisting and pivoting. The radiological score was not different in the 2 groups. There was no correlation between the size of the defect and the outcome. In each individual patient, the severity of symptoms fluctuated significantly during the follow-up (Shelbourne et al., 2003). Again, there was no information as to whether structural repair of the chondral injury was a prerequisite for the good clinical outcome.

The recent improvement of imaging of the articular cartilage with 3D fast spin-echo, or fat suppressed spoiled gradient-echo MRI has allowed detection of chondral lesions with sensitivity and specificity approaching 95\% and $100 \%$ respectively, when compared to the arthroscopic rating (Broderick et al., 1994; Disler et al., 1995; Recht et al., 1996; Kawahara et al., 1998; Bredella et al., 1999). MRI imaging has therefore allowed monitoring of chondral defects to obtain prospective clinical and structural data in symptomatic and asymptomatic groups. These studies have yielded surprising results.

In a longitudinal study, Ding et al. (2006) reported that $43 \%$ of the subjects without a family history of OA, and $57 \%$ in subjects with a family history of OA (Ding et al., 2005b) have chondral defects detectable by MRI. At 2.3 years follow up, $33 \%$ of all subjects had a worsening of the defects as graded by MRI, 37\% had improvement, and the rest remained stable. A worse outcome was associated with female sex, age, and body mass index at baseline. In separate studies, bone geometry (Davies-Tuck et al., 2008) and features of bone remodelling such as the size of "bone marrow lesions" (Wluka et al., 2008) were found to significantly influence the natural history of JSD. Although factors associated with the reproducibility of the MRI grading may have contributed to the defect variation, in general, measurement error was considered to be very low. Importantly, only $18 \%$ of subjects with a cartilage defect had a history of knee trauma. These data show 3 very important points. Firstly, that chondral defects, including full thickness ones are often asymptomatic; secondly, that the majority of these lesions may not be related to traumatic injury as previously thought; finally, and most importantly, that a number of these lesions can improve (and possibly heal) spontaneously. An important caveat for the interpretation of these data is the relatively short followup, which may be insufficient to discern different long term outcomes in patients who have chondral defects in the absence of OA. Indeed, the presence of chondral defects 
in these patients predicted a rate of loss of cartilage volume assessed by MRI of 2-3\% per annum which was nearly double that in subjects without chondral defects (1-2\% per annum) (Ding et al., 2005a). Since the rate of cartilage loss is an independent predictor of joint replacement in patients with OA (Cicuttini et al., 2004), it is arguable that at least a number of such asymptomatic defects may predispose to osteoarthritis.

The spontaneous healing of chondral defects has been confirmed arthroscopically by Nakamura et al. (2008) in patients with co-existing ACL lesions. In this recent paper, the authors compare the Outerbridge grading of chondral defects complicating ACL rupture at first look arthroscopy, with that observed 6-52 months later. In addition, this study revealed a location specific propensity to healing, whereby lesion to the femoral condyles were those most likely to heal, whereas healing to the patella-femoral joint or to the tibial plateaus was exceptional. One interesting feature of this study was that a large proportion of the lesions that healed were partial thickness (Outerbridge grade I and II; $69 \%$ at the medial femoral condyle and $88 \%$ to the lateral femoral condyle). This is in keeping with previous longitudinal studies in humans (Messner and Maletius, 1996; Ding et al., 2008), but at odds with some animal models in which partial thickness defects fail to heal spontaneously (Mankin, 1982) (see below). Besides important differences related to species specificity and location of the lesion, one possible explanation is that experimental lesions are often induced by very sharp instruments that do not induce an injury response which is as strong as that occurring in natural injuries or following blunt trauma (Redman et al., 2004), and, therefore, may be insufficient to activate healing responses. This study confirmed the clinical and MR observation that many such lesions heal spontaneously, without the need for specific cartilage repair intervention.

The natural history and consequence of JSDs in established OA joints has also been investigated. DaviesTuck et al. prospectively recorded chondral lesions in a cohort of patients with osteoarthritis (Davies-Tuck et al., 2008). In this cohort, chondral injuries worsened in $81 \%$ of the cases and improved in only $4 \%$ over 2 years. In a similar prospective study, Wluka showed that the presence of cartilage defects in patients with established symptomatic OA was associated with disease severity and was a predictor of joint replacement within 4 years (Wluka et al., 2005). This worse outcome may reflect factors that reduce the intrinsic repair capacity of cartilage, including low-grade inflammation associated with OA or altered joint biomechanics as a consequence of joint deformity, or ligamentous/meniscal injury. We can summarise these data by saying that cartilage defects may be due to acute mechanical injury and can complicate and accelerate the course of OA; however such lesions may be present in otherwise normal knees, where, at least in some cases, they accelerate the physiological rate of cartilage loss that takes place after the age of 40 years. Importantly, particularly in the absence of OA, some of these defects may undergo healing, and, although age, gender, body mass index, the size and the location of the defects significantly and in combination influence progression (Table 1), it is not presently possible to predict the outcome of one individual defect and there is no "threshold" of any of these parameters that determines unequivocally the outcome. Of course some of these risk factors and others such as malalignment may on one hand hamper repair, and on the other predispose for new cartilage lesions.

These considerations have important clinical consequences in deciding whether, when, and how to treat a chondral defect. Owing to the relative paucity of strong experimental data, and despite attempts to rationalise the current therapeutic approaches (Behrens et al., 2004), recommendations and guidelines vary dramatically from country to country, particularly in Europe. Although general common sense would suggest that chronic, symptomatic, isolated defects are a good indication for interventions such as microfracture or autologous chondrocyte implantation, this is not so obvious for acute defects or when there is other joint pathology. As a consequence, the identification of biomarkers for disease subsets and outcome prediction is being actively pursued. If it were possible to predict the outcome of JSDs accurately, this would not only be valuable for the daily clinical practice but would also facilitate patient selection into clinical trials to increase the power of such studies. Indeed, the identification of a subset of patients who are going to progress would avoid the commonly observed floor effect due to a number of patients who might improve spontaneously in the absence of treatment.

Novel biomarkers for targeting JSD outcome are likely to emerge from cellular and molecular discoveries, in in vivo and in vitro models of experimental cartilage injury. Such models have been used as a platform upon which to study the natural progression of experimental joint surface defects, as well as to determine which molecular pathways are involved in such injury responses and which of these might promote tissue repair. Below we will describe some of the studies of experimental cartilage surface injury from basic histological observations that were first made in the $19^{\text {th }}$ century, to more recent studies involving genetically modified mice in which the molecular pathways of the injury response are beginning to be elucidated. Rather than presenting a systematic review of the innumerable variants of each model, already reviewed elsewhere (Mankin, 1982), we will highlight features and aspects that are particularly relevant to the modern research targets including molecular mechanisms and therapeutic target identification.

\section{Experimental joint surface defects}

When charting the morphological and histochemical response of articular cartilage to scarification or other forms of injury in experimental models in vivo, it is possible to measure not only the early response of the cells in the vicinity of the damage, but also the subsequent attempt at tissue repair. From such studies, a distinction can be drawn between the response of the joint to superficial lesions (those that do not breach the integrity of the osteochondral junction), and those that are full thickness (extending into the underlying subchondral bone). Superficial defects lead to an early, intense, but transient reaction in the cartilage surrounding the lesion. This reaction is characterized 


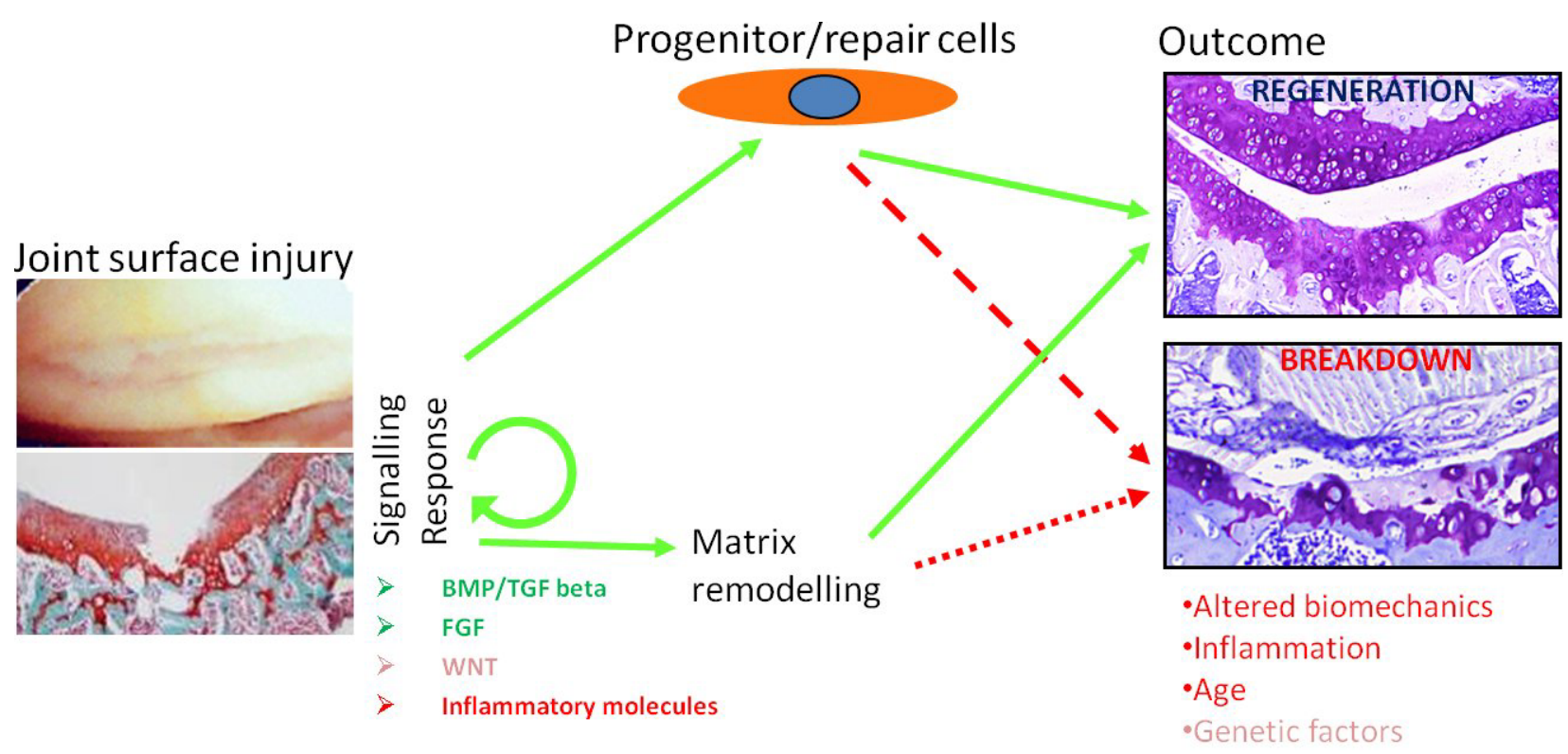

Fig. 1. Acute joint surface injury (e.g., trauma) or chronic mechanical stress (e.g., due to joint malalignment) elicits a molecular response involving several signalling molecules and growth factors. This molecular response activates remodelling and may recruit repair cells, either intrinsic or extrinsic to the joint. Whether this response results in tissue regeneration or breakdown leading to OA likely depends upon other poorly understood contributors including mechanical environment, inflammation, genetic factors and type of cartilage defect e.g., full or partial thickness. In green we have indicated factors presumed to promote a favourable outcome, in red factors that impede it, and in brown factors that are likely to influence the repair responses in different ways, depending on circumstances.

initially by chondrocyte death, and then by a wave of proliferation leading to clustering and intense extracellular matrix production and simultaneous degradation (Mankin, 1982). The efficiency of repair of these lesions is variable (Calandruccio and Glimer, 1962) perhaps in part reflecting differences in experimental conditions, but superficial lesions seldom heal, nor do they evolve into a condition resembling OA (Fisher, 1923; Shands, 1931; Bennett et al., 1932; Calandruccio and Glimer, 1962; Redfern, 1969; Redfern, 1969; Mankin, 1982; Shapiro et al., 1993; Wei et al., 1997). Full thickness defects also stimulate a similar response from the cartilage tissue itself, but in contrast, are more likely to stimulate in addition, an extrinsic repair response, which appears to originate from a fibrous clot at the base of the osteochondral lesion (Meachim, 1963). Others have subsequently studied the origin of these repair cells. Shapiro et al. (1993) generated small osteochondral defects in young rabbit cartilage, which were filled by mesenchymal cells within 2 weeks. Using tritiated thymidine pulse chase experiments they showed labelling first in the bone marrow underlying the defect and subsequently in the repair tissue, but not in the adjacent "healthy" cartilage. The authors concluded that the cells contributing to the repair tissue were derived from the bone marrow (Shapiro et al., 1993). Subsequently, others have identified potential repair cells in other tissues of the joint such as the synovium (Hunziker and Rosenberg, 1996; De Bari et al., 2001a; De Bari et al., 2001b;) and the articular cartilage itself (Dell'accio et al., 2003; Dowthwaite et al., 2004). Ankaru et al. performed a detailed characterization of the early events of repair of full thickness defects in rats (Anraku et al., 2009). This analysis revealed a striking similarity between the patterning and morphogenetic events taking place during repair and those observed during embryonic joint morphogenesis and endochondral bone formation (Karsenty and Wagner, 2002), perhaps explaining why allelic variants of genes playing a role in embryonic skeletogenesis predispose to $\mathrm{OA}$ and are regulated in adult articular cartilage following mechanical injury (Dell'accio et al., 2008).

The nature of the repair tissue has also been studied in some detail. Features of both hyaline and fibrocartilagenous cartilage may be present in the repaired lesion (Bennett et al., 1932; Shands, 1931), and this doubtless influences the long term outcome. Indeed where studies have been extended beyond the first few months, such lesions frequently display features of OA, with chondrocyte clustering, depletion of interterritorial proteoglycans and increased proteoglycans around individual cells (Campbell, 1969; Mitchell and Shepard, 1976). The conclusions of many of these early studies were that (i) injury causes strong activation of chondrocytes, (ii) there is some attempt at repair, especially when the osteochondral junction is breeched, and (iii) that this repair is often fibrocartilagenous and most likely from cells extrinsic to the cartilage e.g. derived from bone marrow or other tissues (Campbell, 1969; Mankin, 1982).

\section{Studying molecular pathways of cartilage injury responses}

The development of in vitro models of cartilage injury has helped to dissect the molecular response of adult articular cartilage to mechanical injury. It is hypothesized that some of the pathways and genes modulated by injury may function to activate repair processes (Fig. 1), and could 


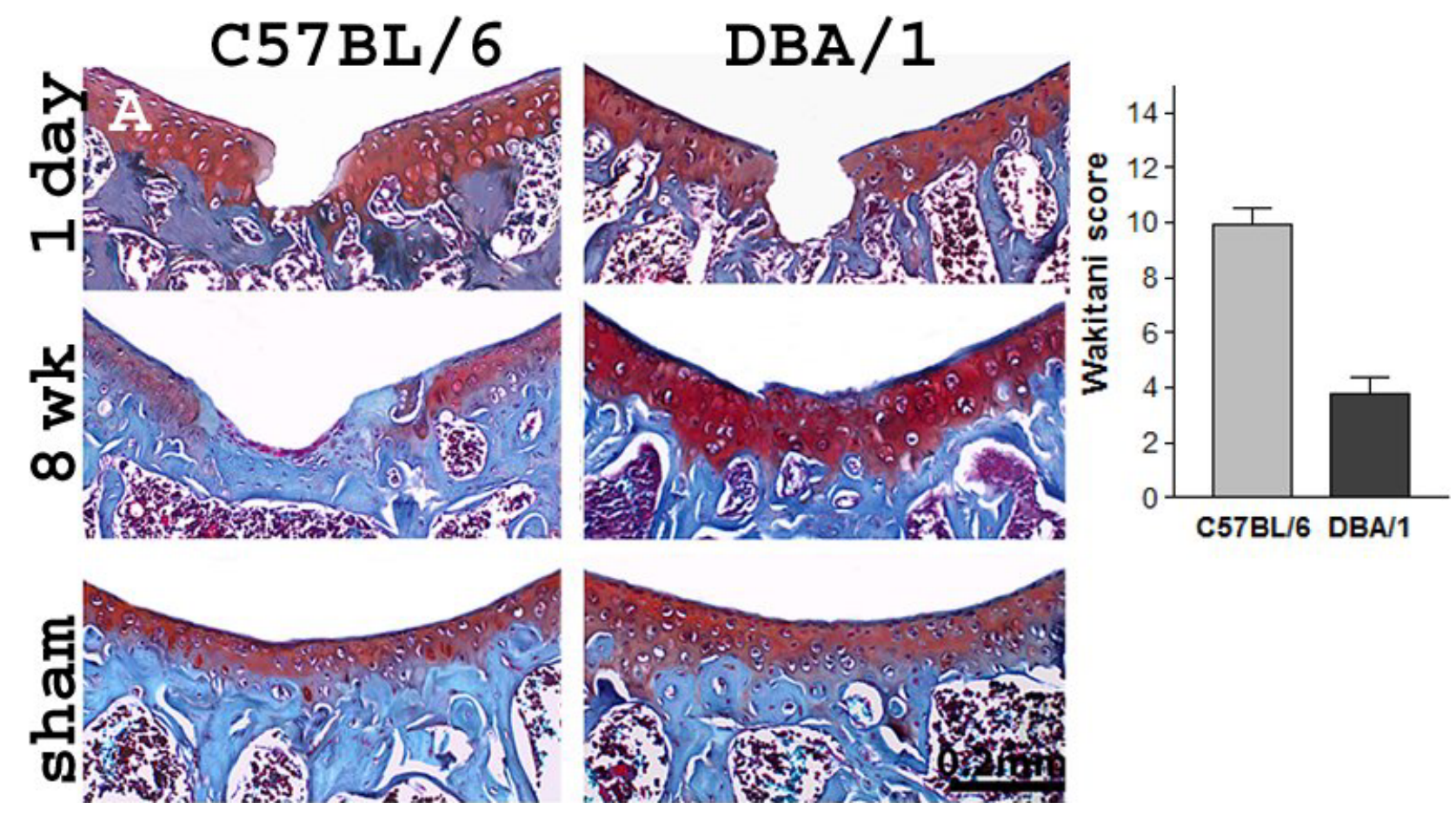

Fig. 2. A controlled full thickness joint surface defect acute mechanical injury to the patellar groove of young-adult mice heals spontaneously in the DBA/1 strain but not in the C57BL/6, thereby demonstrating that there is a genetic component to cartilage healing. On the right, a semi-quantitative repair score. A higher score represents a worse repair outcome. Adapted from (Eltawil et al., 2009), with permission of the publisher.

therefore represent novel therapeutic targets. The converse is also the case that some pathways may promote catabolic activities leading to tissue degradation, and could be negatively targeted to prevent progression to osteoarthritis. From in vitro studies a number of pathways have been identified as being key to the cartilage injury response. These include FGF2 (Vincent et al., 2002), BMPs (Lories and Luyten, 2005) and Wnts (Dell'accio et al., 2006; Dell'accio et al., 2008). Although the function of these pathways specifically in joint surface healing have yet to be tested in vivo in models of cell surface injury, some of them have been studied in models of chronic cartilage injury such as that induced by destabilization of the medial meniscus (DMM) (Glasson, 2007, for review). Using this model, Chia et al. were able to confirm the chondroprotective effect of FGF2 in vivo, by showing that FGF2 null mice develop accelerated disease following surgical induction of OA (Chia et al., 2009). Such models, where injury is continuous (due to joint destabilisation) are complex, because they potentially observe both degradation as well as repair occurring at the same time. Eltawil et al. (2009) recently reported on a novel model of joint surface injury in young adult mice, which specifically observes full thickness cartilage injury responses. In this model, a controlled and reproducible full thickness injury is generated in the patellar groove in an open knee procedure. The cartilage was examined histologically after eight weeks. Their results revealed that young-adult DBA/ 1 mice consistently healed the joint surface defect whereas age-matched $\mathrm{C} 57 \mathrm{BL} / 6$ mice failed to repair and developed features of OA such as proteoglycan loss and surface fibrillation, in the cartilage surrounding the lesion (Figure
2). Interestingly, aged DBA/1 mice failed to repair, but did not develop OA, thereby on one hand confirming the age-dependent efficiency of repair reported in humans (Ding et al., 2007) and animal models (Mankin, 1982; Wei et al., 1997). The different outcome was associated with a specific pattern of tissue responses involving apoptosis, proliferation and matrix remodelling (Eltawil et al., 2009) Such MMP-mediated remodelling has also been observed in focal cartilage defects in larger animals (Hembry et al., 2001). The strain variability demonstrates that, at least in mice, there is a genetic contribution to cartilage repair responses and a specific pattern of molecular events following injury that is associated with efficient repair. The obvious and great advantage of this model over historical injury models is that such studies can be performed in genetically modified mice and thus directly address the role of specific pathways and molecules in successful cartilage repair.

\section{Concluding Remarks}

Joint surface defects are common and may be disabling. The recent explosion of cell based therapies and the advent of novel accurate cartilage imaging techniques has allowed the natural progression of these lesions to be studied. Such prospective studies have revealed that, contrary to what was previously thought, a percentage of JSDs actually heal spontaneously. This is especially the case for superficial lesions and those in otherwise healthy joints. JSDs are much less likely to heal in OA joints, and their presence is a poor prognostic indicator. The increase in sophisticated 
molecular genetic models and tools, in conjunction with suitable in vitro and in vivo model systems is progressively taking us closer to a molecular understanding of repair mechanisms in adult mammals and to the chance of take this knowledge to clinical fruition.

\section{Acknowledgements}

We thank Professor Mary Goldring for critically reviewing the manuscript. Financial support was obtained from the Arthritis Research UK.

\section{References}

Anraku Y, Mizuta H, Sei A, Kudo S, Nakamura E, Senba K, Hiraki Y (2009) Analyses of early events during chondrogenic repair in rat full-thickness articular cartilage defects. J Bone Miner Metab 27: 272-286.

Behrens P, Bosch U, Bruns J, Erggelet C, Esenwein SA, Gaissmaier C, Krackhardt T, Lohnert J, Marlovits S, Meenen NM, Mollenhauer J, Nehrer S, Niethard FU, Noth U, Perka C, Richter W, Schafer D, Schneider U, Steinwachs M, Weise K (2004) [Indications and implementation of recommendations of the working group "Tissue Regeneration and Tissue Substitutes" for autologous chondrocyte transplantation (ACT)]. Z Orthop Ihre Grenzgeb 142: 529-539.

Bennett GA, Bauer W, Maddock SJ (1932) A study of the repair of articular cartilage and the reaction of normal joints of adult dogs to surgically created defects of articular cartilage, 'joint mice' and patellar displacement. Am J Pathol 8: 499-524.

Bredella MA, Tirman PF, Peterfy CG, Zarlingo M, Feller JF, Bost FW, Belzer JP, Wischer TK, Genant HK (1999) Accuracy of T2-weighted fast spin-echo MR imaging with fat saturation in detecting cartilage defects in the knee: comparison with arthroscopy in 130 patients. AJR Am J Roentgenol 172: 1073-1080.

Brittberg M (2008) Autologous chondrocyte implantation - technique and long-term follow-up. Injury 39 Suppl 1: S40-S49.

Brittberg M, Lindahl A, Nilsson A, Ohlsson C, Isaksson O, Peterson L (1994) Treatment of deep cartilage defects in the knee with autologous chondrocyte transplantation. N Engl J Med 331: 889-895.

Brittberg M, Peterson L, Sjogren-Jansson E, Tallheden T, Lindahl A (2003) Articular cartilage engineering with autologous chondrocyte transplantation. A review of recent developments. J Bone Joint Surg Am 85-A Suppl 3: 109115 .

Broderick LS, Turner DA, Renfrew DL, Schnitzer TJ, Huff JP, Harris C (1994) Severity of articular cartilage abnormality in patients with osteoarthritis: evaluation with fast spin-echo MR vs arthroscopy. AJR Am J Roentgenol 162: 99-103.

Calandruccio RA, Glimer WS (1962) Proliferation, regeneration and repair of articular cartilage of immature animals. J Bone Joint Surg 44: 431-455.
Campbell CJ (1969) The healing of cartilage defects. Clin Orthop Relat Res 64: 45-63.

Chia SL, Sawaji Y, Burleigh A, McLean C, Inglis J, Saklatvala J, Vincent T (2009) Fibroblast growth factor 2 is an intrinsic chondroprotective agent that suppresses ADAMTS-5 and delays cartilage degradation in murine osteoarthritis. Arthritis Rheum 60: 2019-2027.

Cicuttini F, Ding C, Wluka A, Davis S, Ebeling PR, Jones G (2005) Association of cartilage defects with loss of knee cartilage in healthy, middle-age adults: a prospective study 26. Arthritis Rheum 52: 2033-2039.

Cicuttini FM, Jones G, Forbes A, Wluka AE (2004) Rate of cartilage loss at two years predicts subsequent total knee arthroplasty: a prospective study. Ann Rheum Dis 63: 1124-1127.

Curl WW, Krome J, Gordon ES, Rushing J, Smith BP, Poehling GG (1997) Cartilage injuries: a review of 31,516 knee arthroscopies. Arthroscopy 13: 456-460.

Davies-Tuck M, Wluka AE, Wang Y, Teichtahl AJ, Jones G, Ding C, Cicuttini FM (2008) The natural history of cartilage defects in people with knee osteoarthritis. Osteoarthritis Cartilage 16: 337-342.

De Bari C, Dell'accio F, Luyten FP (2001a) Human periosteum-derived cells maintain phenotypic stability and chondrogenic potential throughout expansion regardless of donor age. Arthritis Rheum 44: 85-95.

De Bari C, Dell'accio F, Tylzanowski P, Luyten FP (2001b) Multipotent mesenchymal stem cells from adult human synovial membrane. Arthritis Rheum 44: 19281942.

Dell'accio F, Bari CD, Luyten FP (2003) Microenvironment and phenotypic stability specify tissue formation by human articular cartilage-derived cells in vivo. Exp Cell Res 287: 16-27.

Dell'accio F, De Bari C, El Tawil N, Barone F, Mitziadis TA, O'Dowd J, Pitzalis C (2006) Activation of WNT and BMP signaling in adult human articular cartilage following mechanical injury. Arthritis Res Ther 8: R139.

Dell'accio F, De Bari C, Eltawil NM, Vanhummelen P, Pitzalis C (2008) Identification of the molecular response of articular cartilage to injury, by microarray screening: Wnt-16 expression and signaling after injury and in osteoarthritis. Arthritis Rheum 58: 1410-1421.

Ding C, Cicuttini F, Scott F, Boon C, Jones G (2005a) Association of prevalent and incident knee cartilage defects with loss of tibial and patellar cartilage: A longitudinal study. Arthritis Rheum 52: 3918-3927.

Ding C, Cicuttini F, Scott F, Stankovich J, Cooley H, Jones $G$ (2005b) The genetic contribution and relevance of knee cartilage defects: case-control and sib-pair studies. J Rheumatol 32: 1937-1942.

Ding C, Cicuttini F, Scott F, Cooley H, Boon C, Jones G (2006) Natural history of knee cartilage defects and factors affecting change. Arch Intern Med 166: 651-658.

Ding C, Martel-Pelletier J, Pelletier JP, Abram F, Raynauld JP, Cicuttini F, Jones G (2007) Two-year prospective longitudinal study exploring the factors associated with change in femoral cartilage volume in a cohort largely without knee radiographic osteoarthritis. Osteoarthritis Cartilage 16: 443-449. 
Ding C, Cicuttini F, Jones G (2008) How important is MRI for detecting early osteoarthritis?Nat Clin Pract Rheumatol 4: 4-5.

Disler DG, McCauley TR, Wirth CR, Fuchs MD (1995) Detection of knee hyaline cartilage defects using fatsuppressed three-dimensional spoiled gradient-echo MR imaging: comparison with standard MR imaging and correlation with arthroscopy. AJR Am J Roentgenol 165: 377-382.

Dowthwaite GP, Bishop JC, Redman SN, Khan IM, Rooney P, Evans DJR, Haughton L, Bayram Z, Boyer S, Thomson B, Wolfe MS, Archer CW (2004) The surface of articular cartilage contains a progenitor cell population. J Cell Sci 117: 889-897.

Eltawil NM, De BC, Achan P, Pitzalis C, Dell'accio F (2009) A novel in vivo murine model of cartilage regeneration. Age and strain-dependent outcome after joint surface injury. Osteoarthritis Cartilage 17: 695-704.

Fisher AGT (1923) Some researches into the physiological principles underlying the treatment of injuries and diseases of the articulations. Lancet 2: 541 548.

Gelber AC, Hochberg MC, Mead LA, Wang NY, Wigley FM, Klag MJ (2000) Joint injury in young adults and risk for subsequent knee and hip osteoarthritis. Ann Intern Med 133: 321-328.

Glasson SS (2007) In vivo osteoarthritis target validation utilizing genetically-modified mice. Curr Drug Targets 8: 367-376.

Hembry RM, Dyce J, Driesang I, Hunziker EB, Fosang AJ, Tyler JA, Murphy G (2001) Immunolocalization of matrix metalloproteinases in partial-thickness defects in pig articular cartilage. A preliminary report. J Bone Joint Surg Am 83-A: 826-838.

Hjelle K, Solheim E, Strand T, Muri R, Brittberg M (2002) Articular cartilage defects in 1,000 knee arthroscopies. Arthroscopy 18: 730-734.

Hunter W (1743) On the structure and diseases of the articular cartilages. Philos Trans Roy Soc 42B: 514-521.

Hunziker EB, Rosenberg LC (1996) Repair of partialthickness defects in articular cartilage: cell recruitment from the synovial membrane. J Bone Joint Surg Am 78: 721-733.

Karsenty G, Wagner EF (2002) Reaching a genetic and molecular understanding of skeletal development. Dev Cell 2: 389-406.

Kawahara Y, Uetani M, Nakahara N, Doiguchi Y, Nishiguchi M, Futagawa S, Kinoshita Y, Hayashi K (1998) Fast spin-echo MR of the articular cartilage in the osteoarthrotic knee. Correlation of MR and arthroscopic findings. Acta Radiol 39: 120-125.

Lories RJ, Luyten FP (2005) Bone Morphogenetic Protein signaling in joint homeostasis and disease. Cytokine Growth Factor Rev 16: 287-298.

Mankin HJ (1982) The response of articular cartilage to mechanical injury. J Bone Joint Surg Am 64: 460-466.

Meachim G (1963) The effect of scarification on articular cartilage in the rabbit. J Bone Joint Surg 45B: 150-161.
Messner K, Maletius W (1996) The long-term prognosis for severe damage to weight-bearing cartilage in the knee: a 14-year clinical and radiographic follow-up in 28 young athletes. Acta Orthop Scand 67: 165-168.

Mitchell N, Shepard N (1976) The resurfacing of adult rabbit articular cartilage by multiple perforations through the subchondral bone. J Bone Joint Surg Am 58: 230-233.

Morscher E (1979) [Traumatic cartilage lesions in the knee joint] Chirurg 50: 599-604.

Nakamura N, Horibe S, Toritsuka Y, Mitsuoka T, NatsuUme T, Yoneda K, Hamada M, Tanaka Y, Boorman RS, Yoshikawa H, Shino K (2008) The location-specific healing response of damaged articular cartilage after ACL reconstruction: short-term follow-up. Knee Surg Sports Traumatol Arthrosc 16: 843-848.

Peterson L, Minas T, Brittberg M, Nilsson A, SjogrenJansson E, Lindahl A (2000) Two- to 9-year outcome after autologous chondrocyte transplantation of the knee. Clin Orthop 212-234.

Recht MP, Piraino DW, Paletta GA, Schils JP, Belhobek GH (1996) Accuracy of fat-suppressed three-dimensional spoiled gradient-echo FLASH MR imaging in the detection of patellofemoral articular cartilage abnormalities. Radiology 198: 209-212.

Redfern P (1969) On the healing of wounds in articular cartilage. Clin Orthop 64: 4-6.

Redman SN, Dowthwaite GP, Thomson BM, Archer CW (2004) The cellular responses of articular cartilage to sharp and blunt trauma. Osteoarthritis Cartilage 12: 106116.

Shands AR (1931) The regeneration of hyaline cartilage in joints. Arch Surg 22: 178.

Shapiro F, Koide S, Glimcher MJ (1993) Cell origin and differentiation in the repair of full-thickness defects of articular cartilage. J Bone Joint Surg Am 75: 532-553.

Shelbourne KD, Jari S, Gray T (2003) Outcome of untreated traumatic articular cartilage defects of the knee: a natural history study. J Bone Joint Surg Am 85-A Suppl 2: 8-16.

Smith GD, Knutsen G, Richardson JB (2005) A clinical review of cartilage repair techniques. J Bone Joint Surg Br 87: 445-449.

Vincent T, Hermansson M, Bolton M, Wait R, Saklatvala J (2002) Basic FGF mediates an immediate response of articular cartilage to mechanical injury. Proc Natl Acad Sci U S A 99: 8259-8264.

Wei XF, Gao JF, Messner K (1997) Maturationdependent repair of untreated osteochondral defects in the rabbit knee joint. J Biomed Mater Res 34: 63-72.

Wluka AE, Ding C, Jones G, Cicuttini FM (2005) The clinical correlates of articular cartilage defects in symptomatic knee osteoarthritis: a prospective study. Rheumatology (Oxford) 44: 1311-1316.

Wluka AE, Wang Y, Davies-Tuck M, English DR, Giles GG, Cicuttini FM (2008) Bone marrow lesions predict progression of cartilage defects and loss of cartilage volume in healthy middle-aged adults without knee pain over 2 yrs. Rheumatology (Oxford) 47: 1392-1396. 


\section{Discussion with Reviewer}

Reviewer I: From a biological point of view, is there a limiting threshold for a endogenous repair or regeneration, which then would lead to further cartilage degeneration? Authors: The experimental data available, as well as the observational clinical data, suggest that the outcome of regeneration depends on the interaction of different variables including age, body weight, sex, co-morbidity, and genetic background. As a consequence, whereas in animal models, in controlled conditions, changing only one variable at a time, is relatively easy to establish thresholds, in real life it is not currently possible to formulate accurate predictions a priori. For instance, whereas a large number of relatively large JSD were shown to heal in young healthy patients (Ding et al., 2006, text reference), even small lesions very rarely heal in patients with pre-existing osteoarthritis (Davies-Tuck et al., 2008, text reference), or in specific sites such as the patella (Nakamura et al., 2008, text reference). This is the reason why we hope that the identification of biomarkers reflecting the ongoing repair process may represent a significant advance in outcome prediction. Until then, we feel that, particularly for small lesions in otherwise healthy joints, the current practice to clinically monitor JSD and intervene when a chronic course is established is justified. 